\title{
Common knowledge does not have the Beth property
}

\author{
Thomas Studer
}

\begin{abstract}
Common knowledge is an essential notion for coordination among agents. We show that the logic of common knowledge does not have the Beth property and thus it also lacks interpolation. The proof we present is a variant of Maksimova's proof that temporal logics with 'the next' do not have the Beth property. Our result also provides an explanation why it is so difficult to find 'nice' deductive systems for common knowledge.
\end{abstract}

Keywords: Common knowledge, interpolation

\section{Introduction}

Common knowledge and common belief are essential notions for studying coordination among a set of agents. This concerns in particular areas like computer science, logic, game theory, and artificial intelligence. Formalizations of reasoning with and about common knowledge have been widely discussed in the literature, for example in Barwise [3] and in the textbooks Fagin, Halpern, Moses and Vardi [10] as well as Meyer and van der Hoek [22], to give only a few examples.

Halpern and Moses [14] investigate a notion of common knowledge which is based on epistemic logic. They introduce the logic of common knowledge which is based on classical multi-modal logic. In this setting, an agent knowing some proposition $A$ in a state $s$ is usually understood as $A$ holding in all states reachable from $s$ in one step and thus each agent's knowledge may be modeled using a box operator. Furthermore, through arbitrary nesting of boxes epistemic situations of considerable complexity become expressible. However, it is well known that any formula of modal logic can only talk about a finite portion of a model and that this is not sufficient to express certain epistemic situations of particular interest. One such example is common knowledge of a proposition $A$, which can roughly be viewed as the infinitary conjunction all agents know $A$ and all agents know that all agents know $A$ and so on.

Craig interpolation and Beth definability have become traditional questions to ask of a logic system. Let us write $A\left(P_{1}, \ldots, P_{n}\right)$ to indicate that the formula $A$ contains at most the displayed propositional variables $P_{1}, \ldots, P_{n}$ where we 
abbreviate such a sequence of propositional variables by $\vec{P}$. Then the global Beth property (B2) states that for any two propositional variables $X, Y$ and any formula $A(\vec{P}, X)$ if

$$
A(\vec{P}, X), A(\vec{P}, Y) \mid X \leftrightarrow Y,
$$

then there exists a formula $B(\vec{P})$ such that

$$
A(\vec{P}, X) \models X \leftrightarrow B(\vec{P}) .
$$

The Craig interpolation property (CIP) states that if

$$
A(\vec{P}, \vec{Q}) \rightarrow B(\vec{P}, \vec{R})
$$

is valid, then there exists a formula $C(\vec{P})$ such that

$$
A(\vec{P}, \vec{Q}) \rightarrow C(\vec{P}) \text { and } C(\vec{P}) \rightarrow B(\vec{P}, \vec{R})
$$

are valid where $C(\vec{P})$ contains only common propositional variables of $A(\vec{P}, \vec{Q})$ and $B(\vec{P}, \vec{R})$, that is $\vec{P}$ is disjoint from $\vec{Q}$ and $\vec{R}$. Gabbay and Maksimova $[12]$ provide an extensive study of these and related concepts for modal and intuitionistic logics.

Interpolation is important in many areas of computer science, both at the theoretical and at the practical level. Tinelli ${ }^{1}$ for example mentions the following areas of applications:

- Hardware/software specification [4]

- Reasoning with large knowledge bases [2]

- Type inference [17]

- Theorem proving [23]

- Model checking $[15,9]$

The starting point for our work was our cut-elimination result for common knowledge [5]. One of the reviewers for that paper noticed that a nice benefit of a cut elimination theorem like the presence of an interpolation theorem, does not seem to follow, even though this is a major open question.

In this article, we show that the logic of common knowledge does not have the Beth property. Hence, it also lacks interpolation. Our result holds for common knowledge over arbitrary frames as well as over transitive frames. The proof we present is a variant of Maksimova's proof that temporal logics with 'the next' do not have the Beth property [20,19]. A generalization of that proof to fragments of PDL can be found in [18].

\footnotetext{
${ }^{1}$ The Impact of Craig's Interpolation Theorem in Computer Science, talk delivered at Interpolations: A conference in honor of William Craig. Slides available at ftp://ftp.cs. uiowa.edu/pub/tinelli/talks/Craigfest.pdf
} 
The failure of interpolation for common knowledge might be an explanation why it is so difficult to find a 'nice' deductive system for common knowledge. Often the existence of a cut-free system for a logic implies an interpolation property for that logic, see any introduction to proof theory, e.g. [7, 13, 24]. However, if interpolation is a consequence of cut-elimination, then by contraposition we get that the failure of interpolation 'implies' the non-existence of a 'nice' cut-free system. There are indeed several finitary cut-free systems available for common knowledge $[1,16,25]$, but none of them is really satisfactory from a proof-theoretic perspective.

\section{Language and Semantics}

We consider a language with $h$ agents for some finite $h>0$. Propositions $P$ and their negations $\bar{P}$ are atoms. Formulae are denoted by $A, B, C$. They are given by the following grammar

$$
A::=P|\bar{P}| A \wedge A|A \vee A| \square_{i} A\left|\diamond_{i} A\right| \mathrm{C} A \mid \tilde{\mathrm{C}} A
$$

where $1 \leq i \leq h$. The formula $\square_{i} A$ is read as agent $i$ knows $A$ and the formula $C A$ is read as $A$ is common knowledge. The connectives $\square_{i}$ and $C$ have $\diamond_{i}$ and $\tilde{C}$ as their respective duals. The negation $\neg A$ of a formula $A$ is defined as usual by reflecting De Morgan's laws, the law of double negation, and the duality laws for modal operators. We also define $A \rightarrow B:=\neg A \vee B$ and $A \leftrightarrow B:=(A \rightarrow B) \wedge(B \rightarrow A)$. The formula $\mathrm{E} A$ is an abbreviation for everybody knows $A$ :

$$
\mathrm{E} A:=\square_{1} A \wedge \cdots \wedge \square_{h} A \quad \text { and } \quad \tilde{\mathrm{E}} A:=\diamond_{1} A \vee \cdots \vee \diamond_{h} A
$$

Further, we set $\mathrm{C}^{*} A:=\mathrm{C} A \wedge A$.

A Kripke structure $\mathcal{M}$ is a tuple $\left(S, R_{1}, \ldots, R_{h}, \pi\right)$ where $S$ is a non-empty set of so-called possible worlds, each $R_{i}$ is a binary relation on $S$, and $\pi$ is a valuation function assigning to each atomic proposition a set of worlds such that $\pi(\bar{P})=S \backslash \pi(P)$. We write $v \in \mathcal{M}$ for $v \in S$ where $\mathcal{M}=\left(S, R_{1}, \ldots, R_{h}, \pi\right)$.

Given a Kripke structure $\mathcal{M}=\left(S, R_{1} \ldots, R_{h}, \pi\right)$ and worlds $v, w \in S$ we say that $w$ is reachable from $v$ in $n$ steps $(\operatorname{reach}(v, w, n))$ if there exist worlds $s_{0}, \ldots, s_{n}$ such that $s_{0}=v, s_{n}=w$, and for all $0 \leq j \leq n-1$ there exists $1 \leq i \leq h$ with $R_{i}\left(s_{j}, s_{j+1}\right)$. We say $w$ is reachable from $v$ if there exists an $n$ with reach $(v, w, n)$. Moreover, we say $w$ is directly reachable from $v$ if $\operatorname{reach}(v, w, 1)$.

Let $\mathcal{M}=\left(S, R_{1} \ldots, R_{h}, \pi\right)$ be a Kripke structure and $v \in S$ be a possible world. We define the satisfaction relation $\mathcal{M}, v \models A$ inductively on the structure 
of the formula $A$ :

$$
\begin{aligned}
& \mathcal{M}, v \models P \text { if } v \in \pi(P) \quad \text { and } \mathcal{M}, v \models \bar{P} \text { if } v \in \pi(\bar{P}), \\
& \mathcal{M}, v \models A \wedge B \text { if } \mathcal{M}, v=A \text { and } \mathcal{M}, v \models B, \\
& \mathcal{M}, v \models A \vee B \text { if } \mathcal{M}, v=A \text { or } \mathcal{M}, v \models B, \\
& \mathcal{M}, v \models \square_{i} A \text { if } \mathcal{M}, w \models A \text { for all } w \text { such that } R_{i}(v, w), \\
& \mathcal{M}, v \models \diamond_{i} A \text { if } \mathcal{M}, w \models A \text { for some } w \text { with } R_{i}(v, w), \\
& \mathcal{M}, v \models C \text { if } \mathcal{M}, w \models A \text { for all } w \text { such that }(\exists n \geq 1) \operatorname{reach}(v, w, n), \\
& \mathcal{M}, v \models \tilde{C} A \text { if } \mathcal{M}, w \models A \text { for some } w \text { with }(\exists n \geq 1) \operatorname{reach}(v, w, n) .
\end{aligned}
$$

We write $\mathcal{M} \models A$ if $\mathcal{M}, v \models A$ for all $v \in S$. A formula $A$ is called valid if $\mathcal{M}=A$ for all Kripke structures $\mathcal{M}$. Let $\Gamma$ be a set of formulae. We write $\Gamma \models A$ if for all Kripke structures $\mathcal{M}$ such that $\mathcal{M} \models B$ for each $B \in \Gamma$, we also have $\mathcal{M}=A$.

\section{Beth property}

In this section we prove that common knowledge over arbitrary frames does not have the Beth property. If our language includes at least two agents, then the results also holds over transitive frames.

We define the following formulae:

$$
\begin{aligned}
A_{1}(P, X) & :=\mathrm{C}^{*}(\tilde{\mathrm{E}} X \leftrightarrow \bar{X} \wedge \tilde{\mathrm{C}} \bar{P}), & A_{2}(P, X) & :=\mathrm{C}^{*}(X \rightarrow \tilde{\mathrm{C}} \bar{P}), \\
A_{3}(P) & :=\mathrm{C}^{*} \tilde{\mathrm{C} C} P, & A_{4}(X) & :=\mathrm{C}^{*}(\mathrm{E} X \leftrightarrow \tilde{\mathrm{E}} X) .
\end{aligned}
$$

Using these definitions, we set

$$
A(P, X):=A_{1}(P, X) \wedge A_{2}(P, X) \wedge A_{3}(P) \wedge A_{4}(X) .
$$

Lemma 1. Assume that we are given a Kripke structure $\mathcal{M}$ and a world $v \in \mathcal{M}$ such that $\mathcal{M}, v \models A(P, X) \wedge A(P, Y)$. Then the following hold:

1. If $\mathcal{M}, v \models X \wedge \bar{Y}$ or $\mathcal{M}, v \models \bar{X} \wedge Y$, then for all worlds $w$ directly reachable from $v$ we have $\mathcal{M}, w \models \bar{X} \wedge Y$ or $\mathcal{M}, w \models X \wedge \bar{Y}$.

2. Let $n$ be any natural number and let $w$ be a world with $\operatorname{reach}(v, w, n)$. If $\mathcal{M}, v \models X \wedge \bar{Y}$ or $\mathcal{M}, v=\bar{X} \wedge Y$, then $\mathcal{M}, w \models \tilde{C} \bar{P}$.

Proof. 1. Assume $\mathcal{M}, v \models X \wedge \bar{Y}$. From $\mathcal{M}, v \models X$ and $A_{2}(P, X)$ we obtain

$$
\mathcal{M}, v \models \tilde{C} \bar{P} .
$$

Thus by $A_{1}(P, Y)$ we get $\mathcal{M}, v=\tilde{\mathrm{E}} Y$ and then by $A_{4}(Y)$ we conclude

$$
\mathcal{M}, v \models \mathrm{E} Y .
$$

$\mathcal{M}, v \models X,(1)$ and $A_{1}(P, X)$ together yield $\mathcal{M}, v=\neg \tilde{\mathrm{E}} X$ which is

$$
\mathcal{M}, v \mid=\mathrm{E} \bar{X} .
$$

The claim now follows immediately from (2) and (3). If $\mathcal{M}, v \models \bar{X} \wedge Y$, then the dual argument shows the claim. 
2. We show the claim by induction on $n$. Case $n=0$. We have to show $\mathcal{M}, v \models \tilde{C} \bar{P}$ which follows immediately from $\mathcal{M}, v \models X$ and $A_{2}(P, X)$ or from $\mathcal{M}, v \models Y$ and $A_{2}(P, Y)$.

Case $n=k+1$. Let $u$ be a world directly reachable from $v$ such that $\operatorname{reach}(u, w, k)$. By the previous statement we find that $\mathcal{M}, u \models X \wedge \bar{Y}$ or $\mathcal{M}, u \models \bar{X} \wedge Y$. By the induction hypothesis we conclude $\mathcal{M}, w \models \tilde{C} \bar{P}$.

Lemma 2. The following formula is valid: $A(P, X) \wedge A(P, Y) \rightarrow(X \leftrightarrow Y)$.

Proof. Proof by contradiction. Assume that we are given a Kripke structure $\mathcal{M}$ and a world $v \in \mathcal{M}$ such that $\mathcal{M}, v \models A(P, X) \wedge A(P, Y)$. Thus by $A_{3}(P)$ we have

$$
\mathcal{M}, v \models \tilde{\mathrm{C} C} P
$$

Now we suppose $\mathcal{M}, v \models X \wedge \bar{Y}$ or $\mathcal{M}, v \models \bar{X} \wedge Y$ and aim at a contradiction. With the previous lemma we obtain that for all $w$ reachable from $v$ we have $\mathcal{M}, w \models \tilde{C} \bar{P}$. Therefore we have $\mathcal{M}, v \models C \tilde{C} \bar{P}$. This means $\mathcal{M}, v \models \neg \tilde{C} C P$ which contradicts (4).

Lemma 2 implies that $A(P, X)$ defines $X$ implicitly. In the proof of the following theorem we show that there cannot be an explicit definition of $X$. Hence, Beth definability does not hold for the logic of common knowledge.

Theorem 3. The logic of common knowledge does not posses the global Beth property (B2).

Proof. Let $\mathcal{Z}$ be the Kripke model given by:

1. the domain of $\mathcal{Z}$ is the set of integers,

2. the accessibility relations $R_{i}$ for $1 \leq i \leq h$ are given by $R_{i}(u, v)$ if and only if $v=u+1$,

3. $\mathcal{Z}, u \models X$ if and only if $u$ is odd and $u<0$,

4. $\mathcal{Z}, u \models P$ if and only if $u \geq 0$.

We have $\mathcal{Z} \models A(P, X)$.

Let us call a formula $B$ L-stable in $\mathcal{Z}$ if

$$
\exists u \forall v \leq u(\mathcal{Z}, v \models B \Leftrightarrow \mathcal{Z}, u \models B) .
$$

By induction on the structure of formulae we show that every formula $B$ which contains only $P$ as a variable is L-stable in $\mathcal{Z}$.

1. $P$ and $\bar{P}$ are L-stable in $\mathcal{Z}$.

2. $B=C \wedge D$ or $B=C \vee D$. By the induction hypothesis we know that $C$ and $D$ are L-stable in $\mathcal{Z}$, that is there exist $u_{1}, u_{2}$ with

$\forall v \leq u_{1}\left(\mathcal{Z}, v \models C \Leftrightarrow \mathcal{Z}, u_{1} \models C\right) \quad$ and $\quad \forall v \leq u_{2}\left(\mathcal{Z}, v \models C \Leftrightarrow \mathcal{Z}, u_{2} \models D\right)$.

Let $u$ be the minimum of $u_{1}$ and $u_{2}$. Then we have

$$
\exists u \forall v \leq u(\mathcal{Z}, v \models B \Leftrightarrow \mathcal{Z}, u \models B) .
$$


3. $B=\square_{i} C, B=\diamond_{i} C, B=\mathrm{C} C$, or $B=\tilde{\mathrm{C}} C$. By the induction hypothesis we know that $C$ is L-stable in $\mathcal{Z}$, that is there exists $u_{1}$ with

$$
\forall v \leq u_{1}\left(\mathcal{Z}, v \models C \Leftrightarrow \mathcal{Z}, u_{1} \models C\right) .
$$

Let $u=u_{1}-1$. Then we have

$$
\exists u \forall v \leq u(\mathcal{Z}, v \models B \Leftrightarrow \mathcal{Z}, u \models B) .
$$

Therefore, for each formula $B$ which contains only $P$ as a variable, there exists a $u<0$ such that $\mathcal{Z}, u \models B \Leftrightarrow \mathcal{Z}, u-1 \models B$. However, by definition we have $\mathcal{Z}, u \models X \Leftrightarrow \mathcal{Z}, u-1 \not \models X$. Hence $B$ and $X$ must have different truth values either at $u$ or at $u-1$. That means $\mathcal{Z} \not \models B \leftrightarrow X$. Because of $\mathcal{Z} \models A(P, X)$ this implies $A(P, X) \not \models X \leftrightarrow B(\vec{P})$.

Theorem 4. The logic of common knowledge lacks interpolation (CIP).

Proof. Let us introduce another version (B1) of the Beth property: for any formula $A(\vec{P}, X)$ if

$$
\models A(\vec{P}, X) \wedge A(\vec{P}, Y) \rightarrow(X \leftrightarrow Y),
$$

then there exists a formula $B(\vec{P})$ such that

$$
\models A(\vec{P}, X) \rightarrow(X \leftrightarrow B(\vec{P})) .
$$

Lemma 2 and the model in the proof of Theorem 3 show that (B1) does not hold for the logic of common knowledge. We conclude that the logic of common knowledge does not enjoy Craig interpolation (CIP) because (B1) can be derived from $(\mathrm{CIP})$, see $[8,12]$.

A simple adaption of the above arguments yields the following result about common knowledge over transitive frames.

Theorem 5. Assume that our language includes at least two agents. Then the logic of common knowledge over transitive frames does neither have the global Beth property (B2) nor interpolation (CIP).

Proof. Let $\mathcal{Z}$ be the same Kripke model as in the previous proof but replace $R_{i}$ by the following relations:

1. The accessibility relation $R_{1}$ is given by $R_{1}(u, v)$ if and only if $v=u+1$ and $u$ is odd.

2. The accessibility relations $R_{i}$ for $2 \leq i \leq h$ are given by $R_{i}(u, v)$ if and only if $v=u+1$ and $u$ is even.

Again we have $\mathcal{Z} \models A(P, X)$. As before we find that any formula $B$ which contains only $P$ as variable is L-stable in $\mathcal{Z}$ and hence $\mathcal{Z} \not \models B \leftrightarrow X$. 
Note that for this theorem the condition of at least two agents is necessary. $\mathrm{K} 4$ is the basic modal logic (with one agent) over transitive frames. The addition of the common knowledge operator to K4 does not increase the expressive power of K4 since C can be simply defined as $\square$. Gabbay [11] showed that K4 satisfies (CIP). Later Maksimova [21] even proved that every normal extension of K4 has the Beth property (B2).

It is a longstanding open question whether propositional dynamic logic PDL enjoys interpolation, see for instance [18]. At first sight, one could think that our proof can be employed to settle this question in the negative. Of course one easily can encode the common knowledge operator in PDL by making use of union and iteration of programs. Therefore Lemma 2 also holds for PDL. However, Theorem 3 cannot be established for PDL since it is not the case that any PDL formula which contains only $P$ as a variable is L-stable in $\mathcal{Z}$. Consider for instance a formula of the form $\left\langle(a ; a)^{*}\right\rangle C$. This formula states that a state where $C$ holds can be reached in an even number of steps. This is something we cannot express in the language of common knowledge and the fact that we cannot express it is essential for our proof. Therefore, our proof cannot be transfered to PDL.

\section{Conclusion}

Using a variant of Maksimova's proof $[20,19]$ that certain temporal logics do not have the Beth property we have shown that the logics of common knowledge over arbitrary and over transitive frames do not have the Beth property. Hence, they also lack interpolation. However, the problem for other frame conditions remains open. Of particular interest is of course common knowledge over S5, since S5 is usually considered as the logic for modeling knowledge.

As mentioned in the introduction, another major open question is the existence of 'nice' cut-free deductive systems for modal fixed point logics. We believe that the failure of interpolation for common knowledge indicates that there cannot be such a system.

\section{Acknowledgement}

We would like to thank the anonymous referee of [5] for bringing our attention to the problem of interpolation for common knowledge and Larisa Maksimova for pointing us to $[20,19]$. Last but not least we thank the anonymous referees of this paper for the detailed comments they provided.

\section{References}

[1] P. Abate, R. Goré, and F. Widmann. Cut-free single-pass tableaux for the logic of common knowledge. In Workshop on Agents and Deduction, 2007. 
[2] E. Amir and S. McIlraith. Partition-based logical reasoning for first-order and propositional theories. Artif. Intell., 162(1-2):49-88, 2005.

[3] J. Barwise. Three views of common knowledge. In M. Vardi, editor, Theoretical Aspects of Reasoning About Knowledge, pages 365-379. Morgan Kaufman, 1988.

[4] J. Bicarregui, T. Dimitrakos, D. M. Gabbay, and T. S. E. Maibaum. Interpolation in practical formal development. Logic Journal of the IGPL, 9(2), 2001.

[5] K. Brünnler and T. Studer. Syntactic cut-elimination for common knowledge. In Proceedings of Methods for Modalities 5, to appear. Superseded by the journal version [6].

[6] K. Brünnler and T. Studer. Syntactic cut-elimination for common knowledge. Annals of Pure and Applied Logic, to appear.

[7] S. R. Buss. An introduction to proof theory. In S. R. Buss, editor, Handbook of Proof Theory, pages 1-78. Elsevier, 1998.

[8] W. Craig. Three uses of the Herbrand-Gentzen theorem in relating model theory and proof theory. J. Symb. Log., 22(3):269-285, 1957.

[9] J. Esparza, S. Kiefer, and S. Schwoon. Abstraction refinement with Craig interpolation and symbolic pushdown systems. In TACAS '06), pages 489503, 2006.

[10] R. Fagin, J. Y. Halpern, Y. Moses, and M. Y. Vardi. Reasoning about Knowledge. MIT Press, 1995.

[11] D. Gabbay. Craig's interpolation theorem for modal logics. In Conference in Mathematical Logics - London '70, pages 111-127, 1972.

[12] D. M. Gabbay and L. Maksimova. Interpolation and Definability in Modal Logics. Clarendon Press, 2005.

[13] J.-Y. Girard. Proof Theory and Logical Complexity. Bibliopolis, 1987.

[14] J. Y. Halpern and Y. Moses. Knowledge and common knowledge in a distributed environment. Journal of the ACM, 37(3):549-587, 1990.

[15] T. A. Henzinger, R. Jhala, R. Majumdar, and K. L. McMillan. Abstractions from proofs. In Principles of programming languages POPL '04, pages 232 244,2004 .

[16] G. Jäger, M. Kretz, and T. Studer. Cut-free common knowledge. Journal of Applied Logic, 5(4):681-689, 2007.

[17] R. Jhala, R. Majumdar, and R.-G. Xu. State of the union: Type inference via Craig interpolation. In TACAS ' 07 , volume 4424 of $L N C S$, pages 553$567,2007$. 
[18] M. Kracht. Tools and Techniques in Modal Logic. Elsevier, 1999.

[19] L. Maksimova. Temporal logics of "the next" do not have the Beth property. Journal of Applied Non-Classical Logics, 1(1):73-76, 1991.

[20] L. Maksimova. Temporal logics with "the next" operator do not have interpolation or the Beth property. Siberian Mathematical Journal, 32(6):989993, 1991.

[21] L. Maksimova. An analog of Beth's theorem in normal extensions of the modal logic K4. Siberian Mathematical Journal, 33:118-133, 1992.

[22] J.-J. Meyer and W. van der Hoek. Epsitemic Logic for AI and Computer Science. Cambridge University Press, 1995.

[23] G. Nelson and D. C. Oppen. Simplification by cooperating decision procedures. ACM Trans. Program. Lang. Syst., 1(2):245-257, 1979.

[24] A. Troelstra and H. Schwichtenberg. Basic Proof Theory. Cambridge, 1996.

[25] R. Wehbe. A cut-free axiomatization for relativized common knowledge. In Logic Colloquim 2008: Booklet of Abstracts, pages 55-56. University of Bern, 2008.

\section{Address}

Thomas Studer

Institut für Informatik und angewandte Mathematik, Universität Bern

Neubrückstrasse 10, CH-3012 Bern, Switzerland

tstuder@iam.unibe.ch 